\title{
Registros de mamíferos de maior porte na Reserva Florestal do Morro Grande, São Paulo
}

\author{
Mônica de Faria Franco Negrão ${ }^{1}$ e Cláudio Valladares-Pádua ${ }^{2}$ \\ Biota Neotropica v6 (n2)-http://www.biotaneotropica.org.br/v6n2/pt/abstract?article+bn00506022006 \\ Recebido em 17/11/2004 \\ Versão reformulada recebida em 31/3/2005 \\ Publicado em 01/05/2006

\begin{abstract}
${ }^{1}$ Departamento do Patrimônio Genético, Secretaria de Biodiversidade e Florestas, Ministério do Meio Ambiente, SCEN trecho 2 Ed. Sede do Ibama, Bloco G, 70818-900 Brasília, DF (autor para correspondência: monica.negrao@mma.gov.br)

${ }^{2}$ Instituto de Pesquisas Ecológicas, Diretoria - IPÊ, Estrada do Moinho s\n Moinho, 12960-000 Nazaré Paulista, SP
\end{abstract}

\begin{abstract}
Negrão, M.F.F. and Valladares-Pádua, C. Records of mammals of larger size in the Morro Grande Forest Reserve, Sao

Paulo. Biota Neotrop. May/Aug 2006 vol. 6 no. 2, http://www.biotaneotropica.org.br/v6n2/pt/ abstract?article+bn00506022006. ISSN 1676-0603.

Large mammals are known to be important for the maintenance of biological processes and diversity and to be highly susceptible to extinction in fragmented landscapes. However, there are few studies focusing on the community of large mammals in Atlantic Forest remnants. This study aims at surveying large mammals in a remnant of Atlantic forest near to São Paulo. The studied area is the Morro Grande Forest Reserve (RFMG), with 10.870 ha, located in the municipality of Cotia, SP. Seven areas inside the RFMG were studied with two methodologies: 1) line transect sampling and 2) sand plots for footprint recording. Others records of mammal species were also considered. Eighteen species of seven orders were found in the Reserve and were classified in four dietary categories being eight omnivorous, five herbivore, four frugivores and just one carnivorous. The line transect sampling recorded six species of three orders and footprint sampling recorded seven species of five orders. The smaller species, as the squirrel (Sciurus aestuans), the opossum (Didelphis aurita) and the brazilian rabbit (Sylvilagus brasiliense), were the most frequently recorded, and they were present in almost all the study's areas. The prevalence of smaller species and, the presence of some generalist species, indicate the high degree of disturbance and the low quality of this Reserve. The Reserva Florestal do Morro Grande do not maintain the integrity of large mammal community. Key words: mammal community, Atlantic forest, forest fragmentation
\end{abstract}

\section{Resumo}

Negrão, M.F.F. and Valladares-Pádua, C. Registros de mamíferos de maior porte na Reserva Florestal do Morro Grande, São Paulo. Biota Neotrop. May/Aug 2006 vol. 6 no. 2, http://www.biotaneotropica.org.br/v6n2/pt/ abstract?article+bn00506022006. ISSN 1676-0603.

Poucos são os estudos sobre comunidades de mamíferos de maior porte em remanescentes de Mata Atlântica, apesar do importante papel deste grupo na manutenção da diversidade e de processos biológicos e da sua susceptibilidade à extinção em paisagens fragmentadas. O presente trabalho tem como objetivo o levantamento de espécies de mamíferos de maior porte presentes em um remanescente de Mata Atlântica próxima à cidade de São Paulo. O remanescente estudado é a Reserva Florestal do Morro Grande, com área de 10.870 ha, localizada no município de Cotia, SP. Sete áreas da Reserva foram amostradas utilizando duas metodologias: 1) amostragem em transecto linear e 2) parcelas de areia como armadilhas de pegadas. Outros registros diretos e indiretos da ocorrência de espécies do grupo também foram considerados. Foram observadas dezoito espécies, de sete ordens, classificadas em quatro categorias de dieta, sendo oito onívoras, cinco herbívoras, quatro frugívoras e apenas uma carnívora. O método de amostragem em transecto linear amostrou seis espécies de três ordens e as parcelas de areia registraram sete espécies de cinco ordens. As espécies menores, como o esquilo (Sciurus aestuans), o gambá (Didelphis aurita) e o tapiti (Sylvilagus brasiliense) foram as mais registradas e estavam presentes em quase todas as áreas amostradas. O predomínio de espécies menores e de espécies generalistas é um indício do alto grau de perturbação e do comprometimento da qualidade desta Reserva. Conclui-se que a Reserva Florestal do Morro Grande não é capaz de preservar a integridade da fauna de mamíferos de maior porte.

Palavras-chave: comunidade de mamíferos, fragmentação do habitat, conservação, Mata Atlântica 


\section{Introdução}

A conservação da Mata Atlântica enfrenta grandes desafios. Seus domínios abrigam 70\% da população humana brasileira e concentram as maiores cidades e pólos industriais do país (Ministério do Meio Ambiente 2002). Sua área geográfica inicial cobria $12 \%$ do território brasileiro, hoje, restam menos de $8 \%$ de sua extensão original que, mesmo encontrando-se em situação crítica, ainda abriga altos índices de diversidade e endemismo (SOS Mata Atlântica, INPE 1993). Em relação à mastofauna, a Mata Atlântica possui 73 espécies endêmicas (Fonseca et al.1999), 39 ameaçadas de extinção, estando 24 presentes no estado de São Paulo (Ministério do Meio Ambiente http://www.mma.gov.br/port/sbf/index.cfm).

Trabalhos realizados em remanescentes de Mata Atlântica mostram que algumas modificações das comunidades de mamíferos de maior porte estão relacionadas ao tamanho e à disponibilidade de recursos (Chiarello 1999, 2000). Apesar da maioria dos remanescentes de Mata Atlântica serem pequenos e dos mamíferos de maior porte estarem entre os grupos mais susceptíveis à extinção em paisagens fragmentadas são poucos os estudos que abordam este tema (Turner \& Corlett 1995, Primack \& Rodrigues 2001).

Sabe-se, também, que a abundância das espécies deste grupo é bastante afetada por pressões antrópicas como a caça. Vários trabalhos mencionam esta prática como um fator chave em extinções locais de mamíferos e de outras classes (Redford 1997, Carrillo et al.2000, Cullen Jr. et.al 2000, 2001, Escamilla et al.2000). A caça torna-se ainda mais nociva para espécies de ciclos de vida longos e com baixas taxas intrínsecas de crescimento (Bodmer et al.1997).

Estudos como os de Terborgh (1988, 1992), Dirzo \& Miranda (1990), e Janson \& Emmons (1990) mostram a importância dos mamíferos de maior porte na preservação dos sistemas biológicos em florestas tropicais. No entanto, ainda existe uma carência de informações sobre distribuição das espécies e levantamentos da fauna de mamíferos em remanescentes de Mata Atlântica. Este trabalho tem como objetivo o levantamento de espécies de mamíferos de maior porte presentes em um remanescente de Mata Atlântica próximo à cidade de São Paulo.

\section{Material e Métodos}

\section{1. Área de estudo}

A Reserva Florestal do Morro Grande (RFMG) (Figura 1) foi criada através da Lei Estadual $n^{\circ} 1.914$, de 04 de abril de 1979, com a destinação específica de preservação da flora e da fauna e proteção aos mananciais. A RFMG é um dos maiores remanescentes florestais do Planalto Atlântico, abrange uma área de cerca de 10.870 ha, e está localizada no

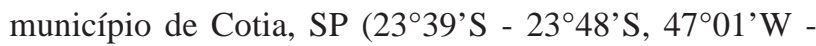
$46^{\circ} 55^{\prime} \mathrm{W}$ ), em uma região que foi submetida a fortes pressões de desmatamento, tanto para agricultura quanto para a exploração de lenha e carvão, e, mais recentemente, para expansão imobiliária.

O clima da região pode ser caracterizado como $C f a$ (Köppen 1948), temperado quente e úmido, sem uma estação de seca bem definida. A precipitação anual média é de 1339 mm, sendo que a média do mês mais seco oscila entre 30 e 60 $\mathrm{mm}$, enquanto a do mês mais quente varia de 150 a $200 \mathrm{~mm}$. O clima predominante no interior da Reserva é o $C f b$ (Köppen 1948), com temperaturas médias mensais que variam de $12,9^{\circ} \mathrm{C}$, em julho, a $21,1^{\circ} \mathrm{C}$, em fevereiro. Por estar em altitudes que variam de 860 a 1075 metros, o clima torna-se particularmente frio, e a ocorrência de neblina e de vento são freqüentes.

A Reserva é atualmente composta por um mosaico de florestas secundárias (sensu Brow \& Lugo 1990) em diferentes estágios de sucessão, algumas com aproximadamente 70-80 anos de regeneração, e outras mais antigas e bem estruturadas. A cobertura florestal predominante do Morro Grande é a Floresta Ombrófila Densa Montana (Veloso et al. 1991), com contribuições da Floresta Estacional Semidecidual e das Florestas Mistas com Araucária, todas pertencentes ao domínio da Mata Atlântica.

Existem duas estradas de terra na parte norte que dão acesso à represa Pedro Beicht, além da Ferrovia FEPASA na parte sul, cortando a Reserva sentido leste/oeste, próxima ao lago da represa (Figura 1). A proximidade da Reserva a cidades e a existência destes acessos à represa contribuem para a entrada de moradores locais, que a utilizam para pesca e lazer, e de animais domésticos.

\section{Metodologias de amostragem}

O levantamento de mamíferos de maior porte foi realizado utilizando duas metodologias complementares em sete áreas dentro da Reserva Florestal do Morro Grande, com diferentes fitofisionomias (Figura 1): 1) amostragem em transecto linear e 2) parcelas de areia como armadilhas de pegadas. A amostragem em transecto linear foi realizada durante os meses de setembro, outubro e novembro de 2001 e fevereiro e março de 2002, em cinco áreas da Reserva. As armadilhas de pegadas foram utilizadas no final do mês de abril e início do mês de maio de 2002 em seis áreas da Reserva.

Para complementar a lista de registros de mamíferos de maior porte na Reserva Florestal do Morro Grande foram considerados outros vestígios diretos ou indiretos da presença de espécies na região, como: fezes, carcaças, rastros, ossadas e visualizações fora da amostragem em transecto linear. 


\subsection{Amostragem em transecto linear}

A metodologia de amostragem em transecto linear, descrita em Buckland et al. (1993) foi empregada devido à sua eficiência comprovada, em especial, para espécies arborícolas como primatas (Peres 1999), mas também pela sua ampla utilização para estimar abundância e densidade de espécies em vários estudos com mamíferos (Emmous 1984, Thompson et al. 1988, Bodmer et al. 1997, Chiarello 1999, 2000, Cullen Jr. et al. 2000, 2001, Carrillo et al. 2000).

Neste trabalho foram realizadas apenas amostragens diurnas com início às seis da manhã e término aproximadamente às seis da tarde. A amostragem resumiuse a explorar a mesma trilha uma ou várias vezes ao dia, com intervalos de 50 min entre cada utilização da mesma. No total, foram percorridos $241 \mathrm{~km}$ de trilhas, a velocidade média constante de aproximadamente $1,3 \mathrm{~km}$ por hora (Tabela 1 ). Inicialmente foram escolhidas cinco trilhas antigas dentro da Reserva (Riacho, Olaria, Trilha C, Ferrovia 2, Quilombo) (Figura 1) freqüentadas por pessoas da região, incluindo caçadores. Porém, não foi possível prosseguir com o trabalho em duas delas (Olaria e Ferrovia 2), por motivos de segurança.

A abundância de espécies foi calculada dividindo o número de indivíduos avistado pelos quilômetros percorridos em cada trilha. No caso de espécies de vida social, a abundância foi calculada dividindo o número de grupos avistados pelos quilômetros percorridos em cada trilha, e multiplicando este valor pelo numero médio de indivíduos presentes em cada grupo avistado.

\subsection{Armadilhas de pegadas}

A utilização de rastros como forma alternativa de registro de mamíferos vem sendo empregada há algum tempo (Thompson et a.l 1988, Janson \& Emmons 1990, Carrillo et al. 2000, Prada 2001). Em 1990, Dirzo \& Miranda apresentaram uma técnica para se trabalhar com rastros em florestas, onde o registro de pegadas é muito precário. Esta técnica baseiase em dispor artificialmente blocos de areia ao longo de trilhas. Apesar desta metodologia não ter sido amplamente testada, ela vem sendo cada vez mais utilizada, mostrandose bastante eficiente para levantamentos de mamíferos em um curto período de tempo. Para trabalhos com espécies terrestres, em locais com baixa densidade de mamíferos, esta técnica pode ser tão, ou mais, eficiente que a metodologia de amostragem em transecto linear (Pardini et al. 2003).

Neste estudo, o método de parcelas de areia foi utilizado de forma padronizada com os trabalhos de Pardini et al. (2003) e Scoss (2002), o que consiste na utilização de uma linha com várias parcelas de areia distantes $10 \mathrm{~m}$ uma da outra. As parcelas correspondem a áreas de 50 por $50 \mathrm{~cm}$ preenchidas com areia fina e úmida até uma altura de aproximadamente $3 \mathrm{~cm}$ e iscadas com um pedaço de banana.
Cada parcela é previamente limpa, retirando-se a vegetação e o folhiço, para colocação da areia em solo limpo. A areia é descompactada e sua superfície homogeneizada para que rastros de animais mais leves sejam registrados.

Em cada uma das seis áreas amostradas (Riacho, Olaria, Trilhas A, B e C e Quilombo) (Figura 1) foram dispostas 20 armadilhas vistoriadas por cinco dias consecutivos, totalizando 100 armadilhas/dia por trilha, e 600 armadilhas/dia para toda a Reserva. A cada dia as armadilhas eram vistoriadas, umedecidas e a isca renovada.

A identificação das pegadas baseou-se em experiência prévia, auxiliada pela utilização do guia de campo de Becker \& Dalponte (1991). Em cada registro de presença foram anotados: a espécie, a data, o local, o horário e o número da parcela. Devido aos registros em cada parcela não apresentarem independência, o que dificulta a utilização deste método para o cálculo de abundância de espécies, calculou-se a freqüência de parcelas com registro de presença das espécies em cada área. Pressupõe-se que os índices indiretos, como pegadas, apresentem relação linear positiva com a abundância relativa das espécies (Wilson et al. 1996).

\section{Análise dos dados}

Além das espécies registradas pelas metodologias aplicadas, outros registros diretos e indiretos foram considerados a fim de obter a riqueza total de mamíferos de maior porte encontradas na Reserva Florestal do Morro Grande. As espécies foram classificadas quanto à dieta e o hábito seguindo Eisenberg \& Redford (1999).

A riqueza de espécies foi estimada pelo procedimento Jackknife1 para os dados obtidos nos dois métodos, para cada uma das áreas amostradas e para toda a Reserva, utilizando o programa EstimateS 6.0b1 (Colwell 2000). O modelo Jackknife é utilizado para minimizar os efeitos de medidas viciadas, como é o caso da riqueza de espécies.

\section{Resultados}

\section{Comunidade de mamíferos de maior porte na Reserva Florestal do Morro Grande}

Considerando todos os tipos de registros, mesmo aqueles obtidos fora das amostragens em transecto linear e parcelas de areia, foram listadas 18 espécies de mamíferos de maior porte na Reserva Florestal do Morro Grande, pertencentes a sete ordens diferentes (um Didelphimorphia, dois Edentata, três Primates, cinco Carnivora, um Artiodactyla, quatro Rodentia e dois Lagomorpha) e enquadradas em quatro categorias de dieta (oito espécies onívoras, cinco herbívoras, quatro frugívoras e apenas uma carnívora - Tabela 2). 


\section{Riqueza de mamíferos de maior porte na} Reserva Florestal do Morro Grande

Durante a amostragem em transecto linear, foram registradas seis espécies de mamíferos de maior porte de três ordens (três Primates, dois Carnivora e um Rodentia) e estimadas oito espécies na Reserva do Morro Grande (Figura 2). O número de espécies variou de zero a três espécies observadas e de zero a 3,96 espécies estimadas por área de amostragem na Reserva.

Utilizando a metodologia de parcelas de areia, foram registradas sete espécies de cinco diferentes ordens (um Didelphimorphia, um Edentata, três Carnivora, um Rodentia e um Lagomorpha) e estimadas 8,6 espécies na Reserva Florestal do Morro Grande (Figura 2). O número de espécies variou de uma a quatro espécies observadas e uma a 5,6 espécies estimadas por área de amostragem na Reserva. A riqueza obtida por esta metodologia foi menor do que os valores registrados no trabalho de Pardini et. al (2003) em três áreas de Mata de Planalto no estado de São Paulo, Parque Estadual Morro do Diabo, Fazenda Tucano e Fazenda Mosquito, e no trabalho de Scoss (2002) realizado no Parque Estadual do Rio Doce, Minas Gerais.

\section{Composição da comunidade de mamíferos de maior porte com relação às categorias de dieta}

Com base nas categorias de dieta, a área Quilombo, com vegetação natural avançada, apresentou espécies de mamíferos classificados em três categorias de dieta (33,3\% herbívoro, 16,7\% frugívoro e 50\% onívoro). As demais áreas, com vegetação natural média, passando por áreas de vegetação natural com influência fluvial média avançada apresentaram: espécies pertencentes a duas categorias na Olaria (50\% herbívoro e 50\% onívoro); espécies pertencentes às quatro categorias de dieta na área do Riacho (25\% herbívoro, 25\% frugívoro e $25 \%$ onívoro e 25\% carnívoros); espécies classificadas em duas categorias de dieta nas áreas B e C (33,3\% frugívoro e 66,7\% onívoro, e 20\% frugívoro e $80 \%$ onívoro, respectivamente); e registros apenas de onívoros na trilha A (Figura 3).

Dentre as sete áreas amostradas, os onívoros apareceram nas seis áreas onde houve registros de mamíferos de maior porte. Em quatro destas áreas, os onívoros foram o grupo com maior porcentagem de espécies registradas. Os frugívoros foram registrados em quatro áreas, os herbívoros em três e os carnívoros em uma única área. Na área em que carnívoros foram registrados, as proporções de espécies entre as categorias de dieta forma semelhantes, não havendo o predomínio de onívoros como na maioria das áreas (Figura 3).

As três maiores espécies presentes na Reserva, o veado (Mazama sp.), a capivara (Hydrochaeris hydrochaeris) e o bugio (Alouatta fusca), possuem dieta frugívora/herbívora, herbívora e herbívora/folívora e são espécies capazes de ocupar florestas secundarias ou perturbadas. A presença do bugio pode estar relacionada à provável ausência ou baixa densidade de seus principais predadores: os grandes felinos e a harpia (Harpia harpyja) (Emmous 1984, Janson \& Emmous 1990 e Chiarello 1999) na região.

\section{Abundância e/ou freqüência calculadas para as espécies de mamíferos de maior porte registradas na Reserva Florestal do Morro Grande}

As espécies mais comuns na Reserva Florestal do Morro Grande foram espécies menores, como o esquilo (Sciurus aestuans), o gambá (Didelphis aurita) e o tapiti (Sylvilagus brasilienses), estando presentes em quase todas as áreas amostradas.

As espécies da ordem Carnivora (gato do mato Leopardus sp, irara -Eira barbara e coati - Nasua nasua) foram registradas em poucas áreas amostradas e, a maioria delas, apresentou baixo número de registros na amostragem por transcecto linear e/ou nas parcelas de areia (Tabela 3).

Das espécies registradas pelo método de transecto linear, o esquilo (Sciurus aestuans) foi a mais abundante na Reserva (Tabela 3). Das espécies registradas nas parcelas de areia, o gambá (Didelphis aurita) apresentou o maior número de registros na Reserva (Tabela 3).

\section{Discussão}

A Reserva Florestal do Morro Grande foi criada com o intuito de proteger as nascentes e cursos d’água formadores do rio Cotia. Desta forma, garantiu-se a preservação de uma área de mata muito próxima à capital paulista, uma das maiores cidades do mundo. Em 1994, a Reserva foi também inserida como área-núcleo na Reserva da Biosfera do Cinturão Verde da Cidade de São Paulo, recebendo assim um reconhecimento internacional pelos significativos serviços ambientais que ela propicia à cidade de São Paulo (Victor et al. 1998). No entanto, se não forem mantidas as suas características e composição originais, a preservação dessas matas não irá garantir a manutenção de todos os seus serviços biológicos.

Apesar da lista de espécies de mamíferos de maior porte registrados na Reserva Florestal do Morro Grande ser extensa, o número de espécies registradas pela amostragem em transecto linear na Reserva Florestal do Morro Grande foi menor do que os valores registrados em seis remanescentes de Mata Atlântica estudados por Chiarello (1999) no estado do Espírito Santo, Reserva Florestal de Linhares, Reserva Biológica de Sooretama, Reserva Biológica Córrego do Veado, Reserva Biológica Córrego Grande e dois outros remanescentes menores, Putiri e M7/317, e em cinco áreas estudadas por Cullen Jr. et al. (2000) situadas em Matas de Planalto no estado de São Paulo, Parque Estadual Morro do Diabo, Estação Ecológica 
de Caetetus, Fazenda Mosquito, Fazenda Tucano e Fazenda Rio Claro. Além disto, a comunidade de mamíferos de maior porte desta Reserva Florestal é composta em sua maioria por espécies generalistas e menos sensíveis à presença humana, indicando um alto índice de perturbação da área. As espécies mais comuns na Reserva Florestal do Morro Grande foram espécies menores, como o esquilo (Sciurus aestuans), o gambá (Didelphis aurita) e o tapiti (Sylvilagus brasilienses), estando presentes em quase todas as áreas amostradas.

As espécies da ordem Carnivora (gato do mato Leopardus sp, irara -Eira barbara e coati - Nasua nasua) foram registradas em poucas áreas amostradas e, na maioria delas, apresentaram baixos valores de abundância e freqüência na amostragem por transcecto linear e nas parcelas de areia. Das espécies registradas pelo método de amostragem em transecto linear, o esquilo (Sciurus aestuans) apresentou maior abundância na Reserva, coincidindo com os resultados encontrados por Chiarello (1999) em quatro dos seis fragmentos de Mata Atlântica estudados no Espírito Santo. No entanto, a abundância encontrada para a Reserva Florestal do Morro Grande é quase dez vezes menor do que as registrado nas áreas amostradas por este autor, aproximando-se dos valores de abundância encontrados por Cullen Jr. et.al. (2000).

Das espécies registradas nas parcelas de areia, o gambá (Didelphis aurita) apresentou o maior número de registros na Reserva. Este resultado difere dos dados obtidos nas áreas estudadas por Pardini et al.(2003) no estado de São Paulo, nas quais o gênero Didelphis apresentou a maior freqüência em apenas uma das três áreas estudadas, com valor seis vezes menor do que o valor de freqüência registrado na Reserva Florestal do Morro Grande.

O predomínio desta espécie na Reserva é um indício do alto grau de perturbação da área. Dados de Fonseca (1989) e Fonseca \& Robinson (1990) mostram que espécies do gênero Didelphis são generalistas de habitat e dieta e se adaptam muito bem a áreas onde a fauna de carnívoros esteja depauperada ou eliminada. Nessas situações, espécies do gênero Didelphis tendem a tornar-se dominante, como ocorre na Reserva.

Das dezoito espécies encontradas, podemos verificar espécies exóticas como: a lebre (Lepus capensis) e o micoestrela (Callitrhix penicillata), mais comum ao bioma Cerrado.

A ocorrência natural de C.penicillata é descrita para o noroeste do estado de São Paulo, delimitada pelo início da área de ocorrência da espécie C. aurita, (Auricchio 1995). Apesar de não haver áreas de ocorrência simpátrica entre as espécies do gênero Callithrix (Stevenson \& Rylands 1988), não podemos afirmar se esta espécie foi introduzida na Reserva pelo homem ou se ocorreu de forma natural. A presença de C. penicillata pode representar uma ameaça à C. aurita, espécie registrada na Reserva e presente na lista de espécies ameaçadas na categoria vulnerável, devido à competição por alimentos e, principalmente, pelo risco de hibridização entre as duas espécies.

A presença da lebre, considerada uma espécie exótica no Brasil, foi registrada em inventários de fauna e trabalhos técnicos mais recentes na região sul e no estado de São Paulo (Silva 1994, Rosa 2002, Mazantti (cord.) 2002, Tortato et al. 2004, Lemes et al. 2004). De acordo com a Diretoria Regional Agrícola de Botucatu, a presença desta espécie no estado de São Paulo pode ser atribuída a sua importação por criadores da região de Paraguaçu Paulista, e o seu registro é mais comum nas regiões de Marília, Araçatuba, Sorocaba e norte e noroeste do estado. A ausência de predadores de maior porte pode contribuir para o crescimento populacional desta espécie invasora no interior da Reserva Florestal do Morro Grande.

Além das dezoito espécies silvestres, também foram registradas espécies domésticas como: cachorro, gato e cavalo. Em uma ocasião, foram observados três cachorros domésticos perseguindo um gato do mato (Leopardus $s p$ ) na área do Riacho. A presença de cachorros domésticos no interior da Reserva pode ser atribuída à alta densidade populacional no entorno da Reserva e à entrada de moradores/caçadores com seus animais domésticos através das estradas que cortam a Reserva.

Foi observada também a presença de pessoas utilizando as trilhas retirando madeira. Foram encontradas antigas cevas e poleiros de caçadores nas áreas da Olaria, Riacho, Ferrovia 2 e Trilha C. Na área do Quilombo, podemos observar a retirada ilegal de palmito e a presença de barracas de lona no interior da mata, provavelmente, para servir de abrigo aos responsáveis por esta ação.

A ausência de espécies cinegéticas como os porcosdo-mato (Tayassu sp), a paca (Agouti paca) e a cutia (Dasyprocta sp) e o encontro de armadilhas, cevas, poleiros de caçadores, além da presença de cachorros domésticos, são indicativos de fortes distúrbios de caça no passado e, provavelmente, ainda no presente. A caça intensa na região pode ter contribuído para extinção local desse grupo de mamíferos. Muitos deles são sensíveis ao contato humano, e o simples fato de pessoas e veículos transitarem pela Reserva pode estar comprometendo a ocorrência dessas espécies da região.

Observamos, neste trabalho, que a fauna de mamíferos presentes na Reserva Florestal do Morro Grande já se apresenta bastante alterada como conseqüência do impacto das atividades humanas à sua volta. Nossas observações nos levam a crer que a Reserva Florestal do Morro Grande não será capaz de preservar a integridade da fauna de mamíferos de maior porte. Mesmo mudanças no comportamento das pessoas que vivem próximas a Reserva e dos proprietários dos vários sítios existentes na região provavelmente serão insuficientes para permitir o restabelecimento de elementos e populações viáveis de animais como os grandes mamíferos predadores. 
Para minimizar os impactos já existentes, recomendase a implementação de um plano de manejo para os mamíferos de maior porte presentes nesta Reserva, que inclua a retirada das espécies exóticas e domésticas citadas, e o monitoramento das espécies com ferramentas que incluam a utilização das duas metodologias apresentadas neste trabalho, de forma a aumentar a probabilidade de registro das espécies de diferentes grupos e hábitos.

O aumento da fiscalização na área da Reversa e a inclusão de trabalhos de educação ambiental envolvendo as comunidades locais devem ser implementados como forma de minimizar os distúrbios causados pela entrada e circulação de pessoas nas trilhas e nas represas.

Recomenda-se, também, priorizar ações de conservação na parte sul da Reserva. As características fitofisionômicas apontam esta área como a que apresenta vegetação em estágios naturais mais avançados. Para o grupo dos mamíferos de maior porte, a parte sul apresentou os maiores valores de riqueza de espécies, além de possuir registro de espécies exclusivos nesta área da Reserva.

\section{Agradecimentos}

Agradeço ao Professor Jean Paul Metzger pela oportunidade de realização deste trabalho, à Renata Pardini pelo convite para participar desta série de artigos do Morro Grande, aos demais pesquisadores que trabalharam na Reserva Florestal do Morro Grande pelo apoio durante este trabalho e aos revisores deste artigo. Agradeço, também, ao meu marido, Jorge, meu filho, Felipe, e familiares, pela compreensão, e a todos os amigos. Este trabalho fez parte do projeto temático BIOTA/FAPESP “Conservação da Biodiversidade em Paisagens Fragmentadas no Planalto Atlântico de São Paulo” (processo 99/05123-4).

\section{Referências bibliográficas}

AURICCHIO, P. 1995. Primatas do Brasil. Terras Brasilis. Editora LTDA, São Paulo.

BECKER, M. \& DALPONTE, J.C. 1991. Rastros de mamíferos silvestres brasileiros-um guia de campo. Editora UnB, Brasília.

BODMER, R.E., EISENBERG, J.F. \& REDFORD, K.H. 1997. Hunting and the likelihood of extinction of amazonian mammals. Conserv. Biol. 11:460-466.

BROW, S. \& LUGO, A.E. 1990. Tropical secondary forest. J. Tropl Ecol. 6:1-32.

BUCKLAND, S.T., ANDERSON, D.R., BURNHAM, K.P. \& LAAKE, 1993. Distance 4.0 Distance sample, Estimating abundance of biological population. Champman and Hall, London.

CARRILLO, E., WONG, G. \& CUARÓNS, A.D. 2000. Monitoring mammal population in Costa Rican protected areas under different hunting restrictions. Conserv. Biol. 14:1580-1591.
CHIARELLO, A.G. 1999. Effects of fragmentation of the Atlantic Forest on mammals communities in south-eastern Brazil. Biol. Conserv. 89:71-82.

CHIARELLO, A.G. 2000. Density and population size of mammals remnants of Brazilian Atlantic Forest. Conserv. Biol. 14:649-1657.

COLWELL, R.K. 2000. Estimate S version 6.0 b1. - Statistical estimation of species richness and shared species from sample. University of Connecticut.

CULLEN, JR. L., BODMER, R.E. \& VALLADARES-PÁDUA, C. 2000. Effects of hunting in habitat fragments of Atlantic forest, Brazil. Biol. Conserv. 95:49-56.

CULLEN, JR. L., BODMER, R.E. \& VALLADARES-PÁDUA, C. 2001. Ecological consequences of hunting in Atlantic forest patches, São Paulo, Brasil. Oryx., 35:137-144.

DIRZO, R. \& MIRANDA, A. 1990. Contemporary neotropical defaunatio and forest struture, function, and diversityaequel to John Terborgh. Conserv. Biol. 4:444-447.

EISENBERG, J.F \& REDFORD, .H. 1999. Mammals of neotropics - the central neotropics. The University of Chicago Press, Chicago and London.

EMMONS, L.H. 1984. Geographic variation in densities and diversities of non-flying mammals in Amazon. Biotropica. 163:210-222.

ESCAMILLA, A., SANVICENTE, M., SOSA, M. \& GALINDO-LEAL, C. 2000. Habitat mosaic, Wildlife availability, and hunting in the tropical forest of Calakmul, Mexico. Conserv. Biol. 14:1592-1601.

FONSECA, G.A.B. 1989. Small mammal species diversity in Brazilian tropical primary and secondary forests of different size. Rev. Bras. de Zool. 63:381-422.

FONSECA, G.A.B. \& ROBINSON, J.G. 1990. Forest size and structure: competitive and predatory effects on small mammals communities. Biol. Conserv. 53:265-294.

FONSECA, G.A.B., HERRMANN, G. \& LEITE, Y.L.R. 1999. Macrogeography of brazilian mammals. In Mammals of the neotropics - the central neotropics (J.F. Eisenberg \& K.H. Redford, eds.) The University of Chicago Press, Chicago and London.v.3,p.549-563.

JANSON, C.H. \& EMMONS, L.H. 1990. Ecological struture of the nonflying mammals community at Cocha Cashu biological station, Manu National Park, Peru. In Four neotropical forests (A.H Gentry, ed.) Yale University Press, New Haven-CT, p.314-338.

KÖPPEN, W. 1948. Climatologia Ed. Fondo Cultural Economica, Mexico City.

LEMES, M.R.S, ANDRIOLO, A., PIOVEZAN, U., RAMOS, H.G.C. \& DUARTE, J.M.B. 2004. Registro de mamíferos através de armadilhas de pegada em áreas a ser reflorestada na região oeste do estado de São Paulo. In XXV Congresso Brasileiro de Zoologia: Resumo, (Universidade de Brasília org.) Sociedade Brasileira de Zoologia, Brasília, p.274-275. 
MAZANTTI, L. (coord.) 2002. Relatório anual de atividades, núcleo de educação ambiental - Capão Bonito. centro de monitoramento ambiental da Serra do Itapety - CEMASI, Universidade Braz Cubas UBC, Universidade de Mogi das Cruzes UMC \& VCP florestal S/A - Célula Sul.

MINISTÉRIO DO MEIO AMBIENTE. 2002. Biodiversidade brasileira. avaliação e identificação de áreas e ações prioritárias para conservação, utilização sustentável e repartição de benefícios da biodiversidade brasileira. Ministério do Meio Ambiente/ Secretaria de Biodiversidade e Florestas. Brasília.

MINISTÉRIO DO MEIO AMBIENTE.( http:// www.mma.gov.br/port/sbf/index.cfm)

PARDINI, R., DITT, E.H., CULLEN, L., BASSI, C. \& RUDRAN, R. 2003. Levantamento rápido de mamíferos terrestres de médio e grande porte. In Metodologia de estudos em biologia da conservação e no manejo da vida silvestre. (Jr.L. Cullen, R. Rudran, \& C. ValladaresPádua, org.) Ed. Curitiba: Editora da Universidade Federal do Paraná e Fundação O Boticário de Proteção da Natureza, p.181-201.

PERES, C.A. 1999. General guidelines for standardizing linetransect surveys of tropical forest primates. Neotropical Primates. 7:111-16.

PRADA, M. 2001. Effects of fire on the abundance of large mammalian herbivores in Mato Grosso, Brazil. Mammalia. 65:55-62.

PRIMACK, R.B. \& RODRIGUES, E. 2001. Biologia da conservação. Gráfica e Editora Midiograf. Londrina.

REDFORD, K.H. 1997. A floresta vazia. In Manejo e conservação de vida silvestre no Brasil (C. ValladaresPádua, R.E. Bodmer \& Jr.L. Cullen org.) Publicações avulsas do Mamirauá. Editora Litera Maciel Ltda. Contagem, p.1-22.

ROSA, A.O. 2002. Comparação da diversidade de mamíferos não-voadores em áreas de floresta de restinga e áreas reflorestadas com Pinus elliottii no sul do Brasil. Tese de mestrado, Universidade do Vale do Rio dos Sinos, Porto Alegre.

SCOSS, L.M. 2002. Impacto de estradas sobre mamíferos terrestres: O caso do parque estadual do Rio Doce, Minas Gerais. Tese de mestrado, Universidade Federal de Viçosa, Viçosa.

SILVA, F. 1994. Mamíferos silvestres: Rio Grande do Sul.Publicação Editada da Fundação Zoobotânica do Rio Grande do Sul, Porto Alegre.

STEVENSON, M. F. \& RYLANDS, A. B. 1988. The marmosets, genus Callithrix. In Ecology and behavior of neotropical primates (R.A. Mittermeier, A.B. Rylands, A.F. Coimbra-Filho \& G.B. Fonseca ed.) World Wildlife Fund, Washington, D.C, p.131-222.
SOS MATA ATLÂNTICA \& INPE. 1993. Evolução dos remanescentes florestais e ecossistemas associados ao domínio da Mata Atlântica. SOS Mata Atlântica e Instituto de Pesquisas Espaciais, São Paulo.

TERBORGH, J. 1988. The big things that run the world - A sequel to E.O. Wilson. Conserv. Biol. 2:402-403.

TERBORGH, J. 1992. Mantinence of diversisity in tropical forests. Biotropica. 242 (B):283-292.

THOMPSON, I.D., DAVIDSON, I.J., O‘DONNELL, S. \& BRAZEAU, F. 1988. Use of track transects to measure the relative occurrence of some boreal mammals in uncut forest and regeneration stands. Can.J Zool. 67:1816-1823.

TORTATO, M.A, PIACENTINI, V.Q \& TORTATO, F.R. 2004. Levantamento preliminar de mamíferos de médio e grande porte numa área de floresta de faxinal no Alto Vale do Itajaí, SC. In XXV Congresso Brasileiro de Zoologia: Resumo, (Universidade de Brasília org.) Sociedade Brasileira de Zoologia, Brasília p.229

TURNER, I.M. \& CORLETT, R.T. 1996. The conservation value of small, isolated fragments of lowland tropical rain forest. Tree 11:330-333.

VELOSO, H.P., RANGEL-FILHO, A.L.R. \& LIMA, J.C.A. 1991. Classificação da vegetação brasileira adaptada a um sistema universal . IBGE, Rio de Janeiro.

VICTOR, M.A.M., FERNÁNDEZ, A.D., FERNÁNDEZ, L.V., MONTRIGAUD, M.E.B. D’A., AMAZONAS, M.C. \& SERRANO, O. 1998. A reserva da biosfera do cinturão verde da cidade de São Paulo. In Workshop: plano de gestão para a reserva da biosfera do cinturão verde da cidade de São Paulo. Instituto Florestal, SP, São Paulo.

WILSON, D.E., COLE,F.R., NICHOLS, J.D., RUDRAN, R. \& FOSTER, M.S. 1996. Measuring and monitoring biological diversity: standard methods for mammals. Washington: Smithsonian Press, p.409

Título: Registros de mamíferos de maior porte na Reserva Florestal do Morro Grande, São Paulo.

Autores: Negrão, M.F.F. \& Valladares-Pádua, C.

Biota Neotropica, Vol. 6 ( número 2 ): 2006

http://www.biotaneotropica.org.br/v6n2/pt/ abstract?article+bn00506022006

Recebido em 17/11/2004 - Versão reformulada recebida em 31/3/2005 - Publicado em 01/05/2006

ISSN 1676-0603 


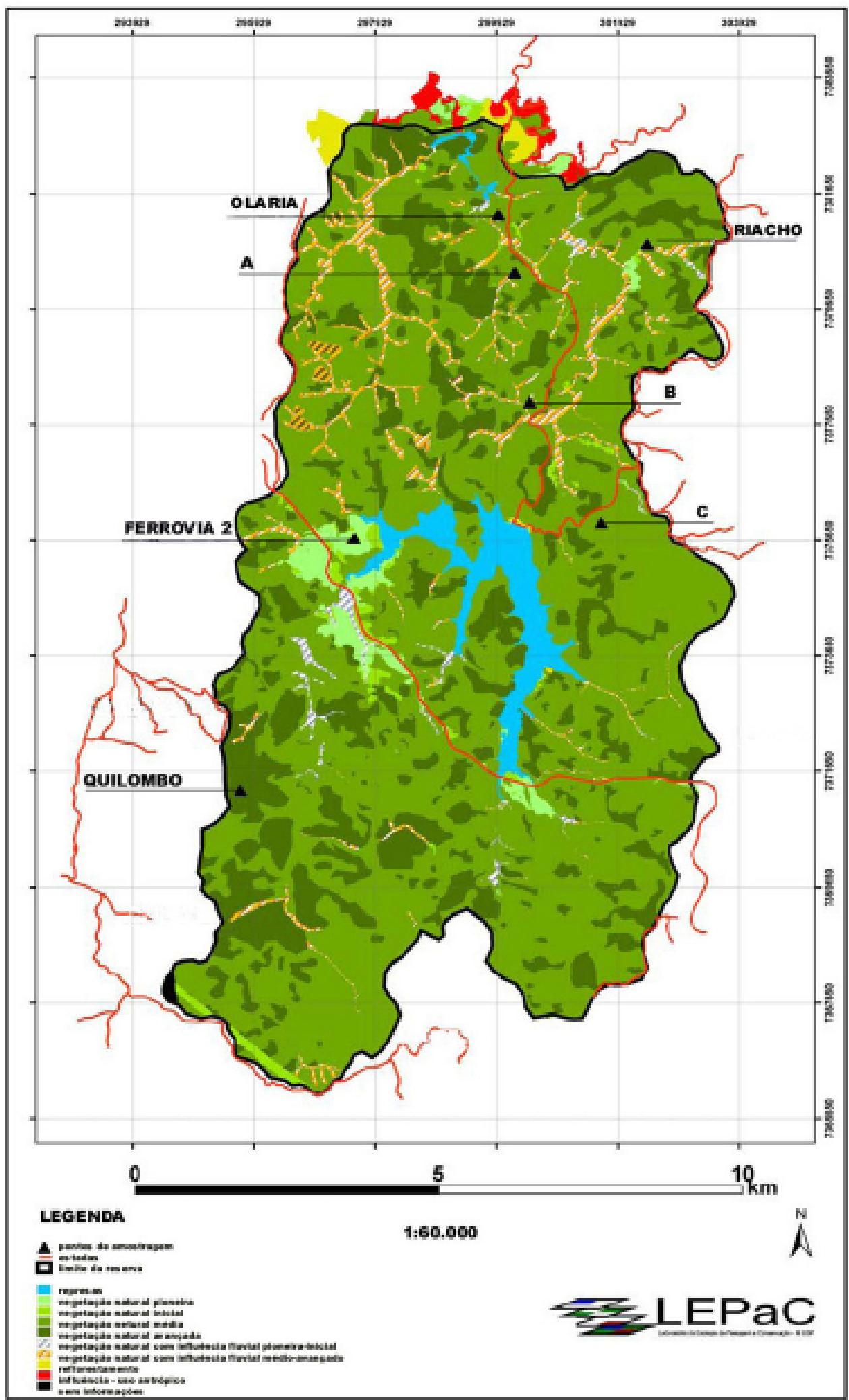

Figura 1: Mapa de vegetação e indicação dos pontos de amostragem dentro da Reserva Florestal do Morro Grande. Figure 1: Map of vegetation and sampling points in the Morro Grande Forest Reserve 


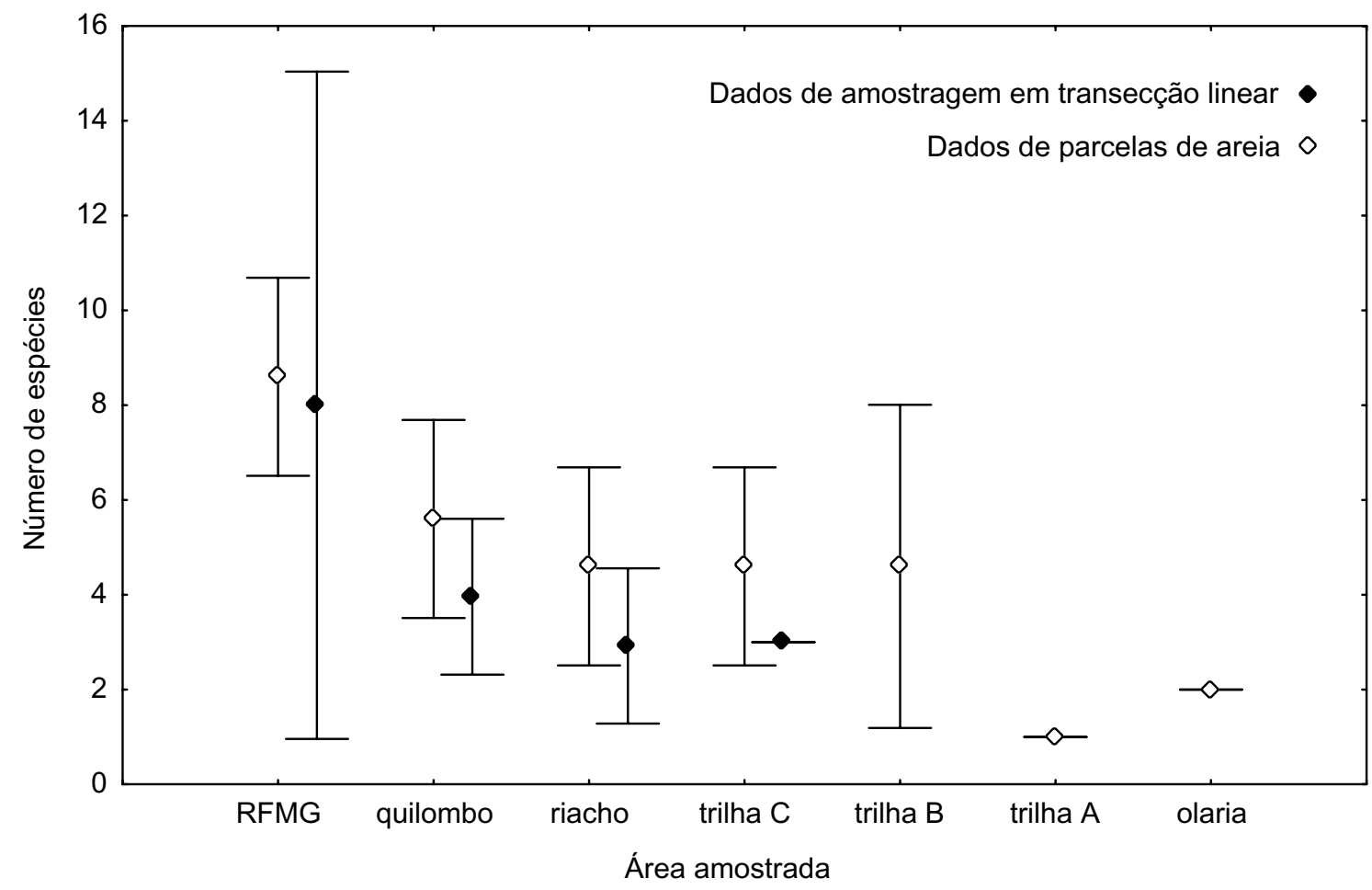

Figura 2: Estimativa de riqueza e intervalos de confiança calculados a partir de 50 randomizações dos dados obtidos, calculados pelo método de Jackknife1, para cada área estudada dentro da Reserva Florestal do Morro Grande onde ocorreu registro de mamíferos de maior porte e para toda a Reserva.

Figure 2: Estimate of relative abundance and confiance limits calculated from 50 randomly choosed data, evaluated by the Jackknife1 method, for each area studied where occurred records of larger size mammals and for the entire Reserve 


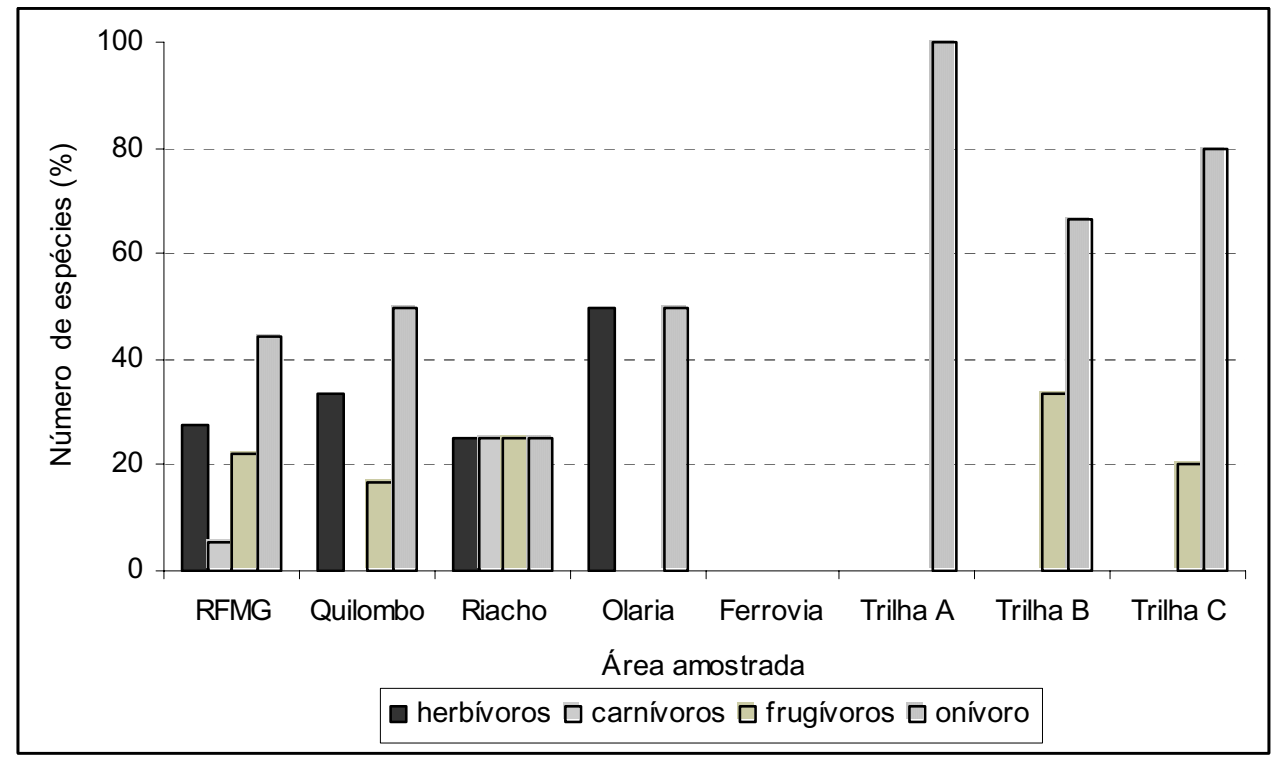

Figura 3: Porcentagem do número registrado de espécies de mamíferos de maior porte por tipo de dieta em cada uma das sete áreas amostradas na Reserva Florestal do Morro Grande, São Paulo.

Figure 3: Percentage of the recorded number of species of larger size mammals for each kind of diet in each one of the seven areas sampled in the Morro Grande Forest Reserve, São Paulo 
Tabela 1: Áreas amostradas, tamanho da trilha utilizada em cada área, número de vezes que cada trilha foi percorrida e quilometragem total percorrida em cada área dentro da Reserva Florestal do Morro Grande durante a amostragem em transecto linear.

Table 1 - Sampled areas, length of the line transect used in each area, number of times in which was walked and total number of km walked in each area within the Morro Grande Forest Reserve, São Paulo

\begin{tabular}{lccc}
\hline Áreas & $\begin{array}{c}\text { Trilha } \\
(\mathbf{k m})\end{array}$ & $\begin{array}{c}\mathbf{N}^{\circ} \mathbf{d e} \\
\text { amostragens }\end{array}$ & $\begin{array}{c}\text { total de } \\
\mathbf{k m}\end{array}$ \\
\hline Quilombo & 2,8 & 24 & 67,2 \\
Riacho & 10 & 6 & 60,0 \\
Olaria & 3,3 & 10 & 33,0 \\
Ferrovia & 2,7 & 4 & 10,8 \\
Trilha C & 3,5 & 20 & 70,0 \\
Total & & 54 & 241,0 \\
\hline
\end{tabular}

http://www.biotaneotropica.org.br 
Tabela 2: Lista das espécies de mamíferos de maior porte registradas na Reserva Florestal do Morro Grande, São Paulo, apresentando o nome comum, a dieta (On - onívoro, Fr - frugívoro, Car - carnívoro, Fol - folhas como principal item da dieta, In - insetos como principal item da dieta) e o hábito (Ter - terrestre, Arb - arborícola, Aqu - aquático) segundo Eisenberg \& Redford (1999), e a forma de registro (c carcaça, $f$ - fezes frescas, o - ossada, $p$ - rastros nas parcelas de areia, $r$ - rastros fora das parcelas de areia, $v$ - visualização durante a amostragem em transecto linear, ob - visualização fora da amostragem em transecto linear).

*espécie ameaçada de extinção

**espécie exótica

Table 2: Listing of the species of larger size recorded in the Reserve and their relation with diet (On $=$ Omnivorous, Fr $=$ Frugivorous, Car $=$ Carnivorous, Fol = leaves as main component of the diet, In = insects as main component of the diet), habit (Ter = Terrestrial, Arb = Arboreal, Aqu = Aquatic and type of recording (according Eisenberg and Redford, 1999), and type of recording (c = carcass, $f=$ fresh feces, $o=b o n e s$, $p=$ footprints on sandy spots, $r=$ footprints out of sandy spots, $v=$ observation during line transect sampling, ob $=$ observation out of the line transect sampling)

*espécie ameaçada de extinção

**espécie exótica

\begin{tabular}{|c|c|c|c|c|}
\hline Táxon & Nome comum & Dieta principal & Hábito & $\begin{array}{l}\text { Tipo de } \\
\text { registro }\end{array}$ \\
\hline
\end{tabular}

\section{Didelphimorphia}

Didelphis aurita

Gambá

On

Ter/Arb

$\mathrm{p}$

\section{Edentata}

Dasypus novemcinctus

Tatu galinha

$\mathrm{On} / \mathrm{In}$

Ter

$\mathrm{p}$

Euphractus sexcinctus

Tatu peba

On

Ter

r

\section{Primates}

Callithrix penicillata**

Callithrix aurita*

Allouata fusca

\section{Carnivora}

Cerdocyon thous

Nasua nasua

Procyon cancrivorus

Eira barbara

Leopardus sp. *

Artiodactyla

Mazama sp.

\section{Rodentia}

Cavia sp.

Hydrochaeris hydrochaeris

Sciurus aestuans

Sphiggurus insidiosus

\section{Lagomorpha}

Sylvilagus brasilienses

Lepus capensis**
Mico estrela

Sagüi escuro da serra

Bugio

Lobinho

Coati

Mão-pelada

Irara

Gato do mato pintado

Veado

Preá

Capivara

Esquilo

Ouriço-cacheiro
On

On

$\mathrm{He} / \mathrm{Fol}$

On

On

$\mathrm{Fr} / \mathrm{On}$

On

Car

$\mathrm{Fr} / \mathrm{He}$

Arb

$\mathrm{V}$

Arb

V

Arb

$\mathrm{V}$

r

$\begin{array}{cc}\text { Ter } & \mathrm{r} \\ \text { Ter/Arb } & \mathrm{v} / \mathrm{p}\end{array}$

Ter

r

Ter/Arb

$\mathrm{p}$

Ter

$\mathrm{v} / \mathrm{p} / \mathrm{f}$

$\mathrm{He}$

Ter

r

$\mathrm{He}$

Fr

Ter/Aqu

$\mathrm{r} / \mathrm{f}$

Arb

$\mathrm{v} / \mathrm{p}$

Fr

Arb

$\mathrm{r} / \mathrm{c}$

http://www.biotaneotropica.org.br

\begin{abstract}
Tapiti
\end{abstract}
Lebrão
$\mathrm{He}$

$\mathrm{He}$
Ter

Ter $\mathrm{p} / \mathrm{ob}$

o 


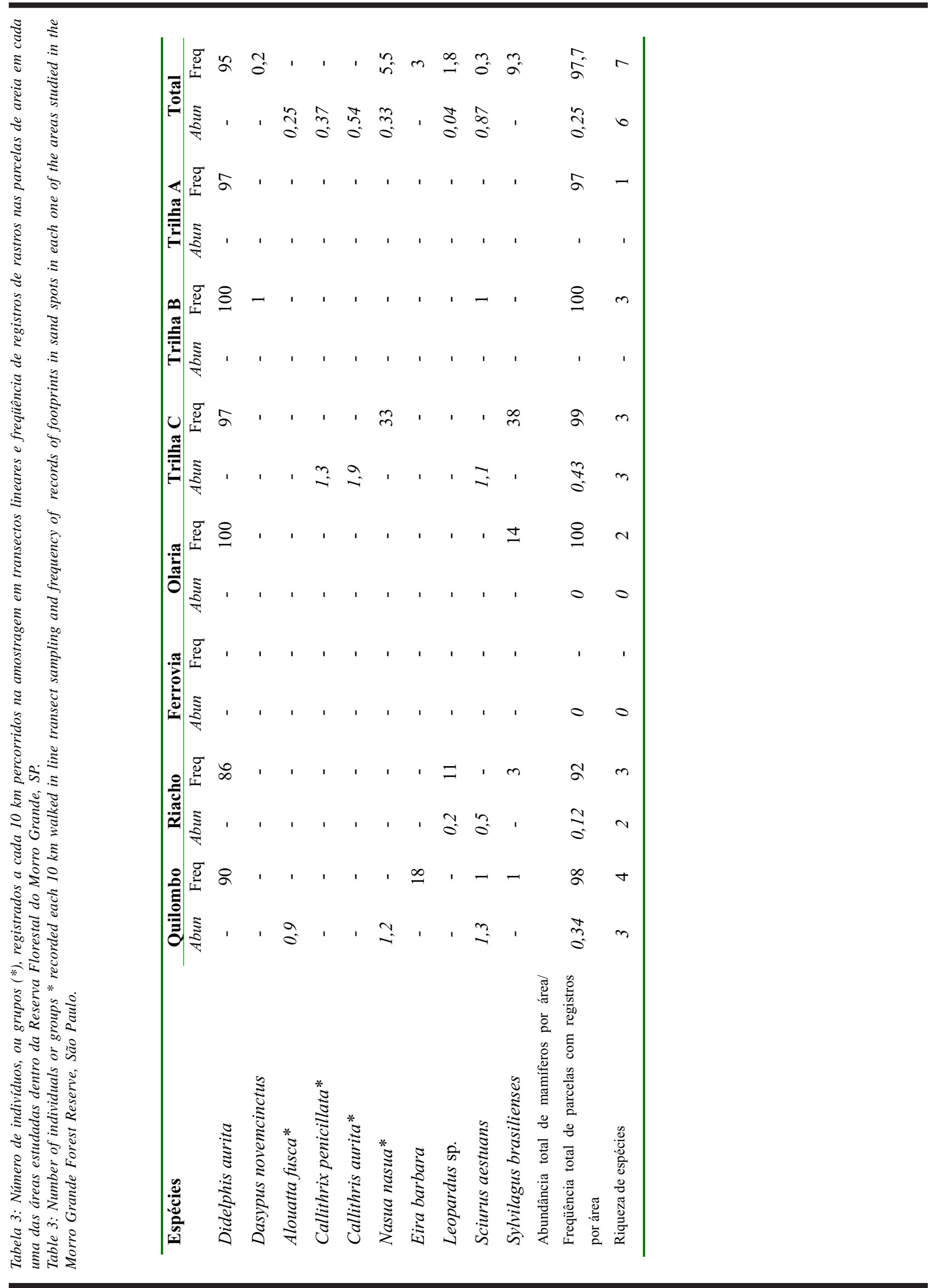

http://www.biotaneotropica.org.br 